\title{
EQUIVALENCE OF SECOND-ORDER ODES TO EQUATIONS OF FIRST PAINLEVÉ EQUATION TYPE
}

\author{
YU.YU. BAGDERINA
}

\begin{abstract}
We consider equivalence problem for equations of a degenerate type, which involve, for example, the first Painlevé equation. In terms of algebraic and differential invariants of the family of equations with the cubic nonlinearity in the first-order derivative, we obtain the necessary condition of equivalence to some equations of this type with a known solution. We prove a criterion of equivalence to the first Painlevé equation under point transformations.
\end{abstract}

Keywords:first Painlevé equation, equivalence, invariant.

Mathematics Subject Classification: 34M55, 34M15

\section{INTRODUCTION}

The problem on equivalence of second order ordinary differential equations (ODEs)

$$
\frac{d^{2} y}{d x^{2}}=S(x, y)\left(\frac{d y}{d x}\right)^{3}+3 R(x, y)\left(\frac{d y}{d x}\right)^{2}+3 Q(x, y) \frac{d y}{d x}+P(x, y)
$$

under point change of variables

$$
z=\xi(x, y), \quad w=\eta(x, y), \quad \frac{\partial(\xi, \eta)}{\partial(x, y)} \neq 0
$$

attracts a lot of attention since the end of XIXth century [1]-[10]. Class of equations (1) is closed w.r.t. transformations (2). It contains 50 equations [11, Ch. 14] obtained in the classification of second order ODEs having no moving critical points except for the poles [12, 13] including six Painlevé equations. The problem of equivalence of a second order ODE to the first Painlevé equation

$$
\frac{d^{2} w}{d z^{2}}=6 w^{2}+z
$$

under point transformations was studied in [10] and [14]-[19]. In [20], there was considered a problem of equivalence of the first Painlevé equation to the generalized Emden-Fowler equation under (non-local) Sundman transformation.

It was proven in [21] that all Painlevé equations can be reduced to the equation

$$
\frac{d^{2} y}{d x^{2}}=f(x, y)
$$

Yu.Yu. Bagderina, Equivalence of Second-order Odes to equations of the first Painlevé EQUATION TYPE.

(C) Bagderina Yu.Yu. 2015.

The research is supported by a grant of Russian Science Foundation (project no. 14-11-00078).

Submitted October 14, 2014. 
by some change (2); canonical form (4) was obtained for third, fourth, fifth, and sixth Painlevé equation. It was also shown in [21] that only special change of variables

$$
\tilde{x}=k \int \phi^{2}(x) d x+x_{0}, \quad \tilde{y}=\phi(x) y+\chi(x), \quad k, x_{0}=\text { const }, \quad k, \phi(x) \neq 0
$$

does not lead equation (4) outside this class.

In the most part of works devoted to the equivalence problem for equations (1) [1]- [5, 8] there was studied only the general (non-degenerate) case for equation (1) described by the relation $J_{0} \neq 0$ (the formula for $J_{0}$ is provided in the next section). For all the Painlevé equations we have $J_{0}=0$ and thus, they belong to degenerate types of equation (1). In [1] there were also studied degenerate cases of equation (1) but their complete classification was not made. Such classification was made in [6, 7] by the methods of differential geometry. Later, in [9, it was made by exterior Cartan forms, and later in [10] by employing Lie's infinitesimal approach. At that, the result applicable to each equation (1) was provided only in works [7, 10]. In [6] the formulae for the invariants of equation (1) were obtained in a special coordinate system and to apply them, one should first make a preliminary change of variables (i.e., requires making several interations). The result of [9] for degenerate types of equation (1) including, in particular, Painlevé equations needs that equation (1) should be first reduced to (4).

Equation (1) of first type in [10] correspond to the general case determined in [6, 7]. Equations of ninth type in [10] coincide with the case of maximal degeneration in [6, 7]. For other seven types of equation (1) introduced in [10], it is to be studied independently to which cases of intermediate degeneration in original classification [6, 7] these types correspond. In the main case the formulae of connection between the invariants of equation (1) constructed in [7] and [10] are provided in [10, Sect. 1]. Similar formulae in the degenerate case which includes five of six Painlevé equations are given in [22, Sect. 7]. The correspondence between the invariants in [10] and those applied in [1, 21, 23] was established in [22, Sect. 3] and [24].

In the present work the equivalence conditions were obtained by employing algebraic (depending on $x, y$ ) invariants of equations (1) constructed in [10]. Equivalent equations have the same sets of (absolute) invariants. According to the classification in [10, equation (3) belongs to the sixth type of equation (1). Other five types of Painlevé equations belong to the fourth type and the equivalence conditions for them were studied in [10, 19, 22, 23, 24]. The present paper is devoted to the equations of sixth type, i.e., to the most degenerate equations (1) possessing invariants. In Section 2 we describe invariants of such equations and we show that by some change of variables (2) all of them are reduced either to

$$
\frac{d^{2} w}{d z^{2}}+\frac{1}{4 w^{3}}+F(z)=0, \quad F(z) \neq 0,
$$

or to

$$
\frac{d^{2} w}{d z^{2}}=6 w^{2}+f(z)
$$

In Sections 3, 4 we calculate the invariants for these equations and obtain necessary conditions of equivalence to equations (4) of sixth type for which in [25] either the general solution was given or the order was reduced. Namely, in Theorems 2-6 we describe five non-equivalent equations of sixth type admitting point symmetries. Four of these equations are integrable and in one of them the order can be reduced. Transformation (2) relating equivalent equations is constructed by employing both algebraic and differential (depending on $x, y, y^{\prime}=d y / d x$ ) invariants of equations (1). It was shown in [26] that in solving the equivalence problem of a second order ODE the maximal amount of differentiations required for constructing the invariants should be made for equation (7) with $\left(f^{-1 / 4}\right)^{\prime \prime} \neq 0$. 
As papers [14]-[19] show, the criterion of equivalence to equation (3) can be obtained by means of various approaches. In Section 5 it is reproduced in Theorem 7 . At the example of the first Painlevé equation we show how to prove the sufficiency for conditions of equivalence to equation (1), once among its algebraic invariants there are invariants $I_{j}, I_{k}$ such that

$$
\frac{\partial\left(I_{j}, I_{k}\right)}{\partial(x, y)} \neq 0 .
$$

Relation (8) expresses the functional independence of invariants $I_{j}$ and $I_{k}$. The approach based on the functional independence of the invariants it known and employed for a long time (see, for instance, [5, P. 7], [17], [18] or formulae (7) in [19]). In [22] this approach was employed in the proof of a criterion of equivalence to the second Painlevé equation and to equation XXXIV in [11] which can be reduced to the second Painlevé equation by a differential substitution. For the other Painlevé equations the sufficiency of equivalence conditions was established if applying of these conditions to ODE (4), up to transformation (5) gives the canonical form of the corresponding Painlevé equation in [21]. In the case of third and fourth Painlevé equations, this approach was employed in works [24] and [22], respectively.

In Section 6 we provide examples of applying differential invariants in constructing transformation (2) relating two equivalent equations with no algebraic invariants satisfying condition (8). We consider the case when all the algebraic invariants of an equation are constant as well as the case when all of them depend on one variable. It is also shown that the generalized Emden-Fowler equation found in [20] is related with the first Painlevé equation by a point change of variables (5).

\section{INVARIANTS FOR EQUATIONS OF SIXTH TYPE}

Here we partially reproduce the classification of second order ODEs in [10, Thms. 2, 7] concerning the equations of sixth type.

Theorem 1. Equation (1) of sixth type are characterized by the relations

$$
J_{0}=0, \quad \beta_{1} \neq 0, \quad j_{0}=0, \quad j_{1}=0, \quad j_{2}=0, \quad j_{3} \neq 0, \quad \Gamma_{0} j_{3} \neq 5 \beta_{1} .
$$

The basis of its differential invariants is

$$
\begin{aligned}
& I_{0}=\frac{1}{j_{3}^{3 / 2}}\left(\frac{15 e_{0}}{I_{1}-5}-\frac{3 y^{\prime}}{\beta_{1}\left(\beta_{1}+y^{\prime} \beta_{2}\right)}\right) \\
& I_{1}=\frac{\Gamma_{0} j_{3}}{\beta_{1}}, \quad I_{2}=\frac{1}{5 j_{3}^{2}}\left(\left(5-I_{1}\right) \Lambda+\frac{4 \Gamma_{0} e_{0}^{2}}{\beta_{1}}\right) .
\end{aligned}
$$

An arbitrary algebraic invariant of equation (1) can be obtained by applying functional-algebraic operations and operators of invariant differentiation

$$
\mathcal{D}_{1}=\frac{1}{j_{3}}\left(\beta_{2} \partial_{x}-\beta_{1} \partial_{y}\right), \quad \mathcal{D}_{2}=\frac{15 e_{0}}{\left(I_{1}-5\right) \sqrt{j_{3}}}\left(\beta_{2} \partial_{x}-\beta_{1} \partial_{y}\right)-\frac{3}{\beta_{1} \sqrt{j_{3}}} \partial_{x}
$$

to $I_{1}, I_{2}$. Algebraic invariants for all the equations of sixth type satisfy trivial relations

$$
\begin{aligned}
& 5 I_{11}+3 I_{1}\left(5-I_{1}\right)=0, \quad 5 I_{12}-2\left(2 I_{1}+15\right) I_{2}+\left(5-I_{1}\right)\left(\frac{46}{45} I_{1}+33\right)=0, \\
& I_{21}=0, \quad \mathcal{D}_{1}\left(\mathcal{D}_{2}^{n} I_{2}\right)=\left(\frac{1}{10}(n+8) I_{1}+\frac{3}{2}(n+4)\right) \mathcal{D}_{2}^{n} I_{2}, \quad n \in \mathbb{N} .
\end{aligned}
$$


In this theorem we employ the notations

$$
I_{i j}=\mathcal{D}_{i} I_{j}, \quad I_{k i j}=\mathcal{D}_{k}\left(\mathcal{D}_{i} I_{j}\right), \quad I_{l k i j}=\mathcal{D}_{l}\left(\mathcal{D}_{k}\left(\mathcal{D}_{i} I_{j}\right)\right), \quad i, j, k, l=1,2,
$$

for the derivatives of the invariants obtained by applying invariant differentiations (11) to $I_{1}$, $I_{2}$. The quantities involved in (9)-(11)

$$
\begin{aligned}
& \Gamma_{0}=3 \beta_{2} \gamma_{10}+\beta_{1}\left(\gamma_{20}-4 \gamma_{11}\right), \quad \Gamma_{1}=\beta_{2}\left(4 \gamma_{20}-\gamma_{11}\right)-3 \beta_{1} \gamma_{21}, \\
& J_{0}=\frac{1}{3}\left(\beta_{2} \Gamma_{0}-\beta_{1} \Gamma_{1}\right), \quad j_{0}=\frac{3}{\beta_{1}}\left(\frac{\beta_{2}}{\beta_{1}} \delta_{10}-\delta_{11}\right)+\frac{6 \gamma_{10}}{\beta_{1}^{2}}\left(\gamma_{11}-\frac{\beta_{2}}{\beta_{1}} \gamma_{10}\right), \\
& j_{1}=\frac{5}{6}\left(2 \beta_{2} \delta_{20}-\beta_{1} \delta_{30}-\frac{\beta_{2}^{2}}{\beta_{1}} \delta_{10}\right)+\left(\gamma_{20}+\gamma_{11}-2 \frac{\beta_{2}}{\beta_{1}} \gamma_{10}\right)\left(\gamma_{20}-\frac{2}{3} \gamma_{11}-\frac{\beta_{2}}{3 \beta_{1}} \gamma_{10}\right), \\
& j_{2}=\frac{1}{\beta_{1}}\left(\delta_{20}-\frac{\beta_{2}}{\beta_{1}} \delta_{10}\right)+\frac{\gamma_{10}}{5 \beta_{1}^{2}}\left(7 \frac{\beta_{2}}{\beta_{1}} \gamma_{10}-6 \gamma_{20}-\gamma_{11}\right), \quad j_{3}=\frac{3}{5}\left(\frac{\delta_{10}}{\beta_{1}^{3}}-\frac{6 \gamma_{10}^{2}}{5 \beta_{1}^{4}}\right), \\
& e_{0}=\frac{\epsilon_{10}}{5 \beta_{1}^{4}}-\frac{21 \gamma_{10} \delta_{10}}{25 \beta_{1}^{5}}+\frac{84 \gamma_{10}^{3}}{125 \beta_{1}^{6}}, \quad \Lambda=\frac{\lambda_{10}}{5 \beta_{1}^{5}}+\frac{4}{25 \beta_{1}^{6}}\left(7 \delta_{10}^{2}-8 \gamma_{10} \epsilon_{10}\right)
\end{aligned}
$$

are calculated by means of relative invariants

$$
\begin{aligned}
& \beta_{1}=\alpha_{1 x}-\alpha_{0 y}+R \alpha_{0}-2 Q \alpha_{1}+P \alpha_{2}, \quad \beta_{2}=\alpha_{2 x}-\alpha_{1 y}+S \alpha_{0}-2 R \alpha_{1}+Q \alpha_{2}, \\
& \gamma_{10}=\beta_{1 x}-Q \beta_{1}+P \beta_{2}, \quad \delta_{10}=\gamma_{10 x}-2 Q \gamma_{10}+P\left(\gamma_{20}+\gamma_{11}\right)-5 \alpha_{0} \beta_{1}, \\
& \gamma_{11}=\beta_{2 x}-R \beta_{1}+Q \beta_{2}, \quad \delta_{11}=\gamma_{11 x}-R \gamma_{10}+P \gamma_{21}-\alpha_{1} \beta_{1}-4 \alpha_{0} \beta_{2}, \\
& \gamma_{20}=\beta_{1 y}-R \beta_{1}+Q \beta_{2}, \quad \delta_{20}=\gamma_{20 x}-R \gamma_{10}+P \gamma_{21}-4 \alpha_{1} \beta_{1}-\alpha_{0} \beta_{2}, \\
& \gamma_{21}=\beta_{2 y}-S \beta_{1}+R \beta_{2}, \quad \delta_{21}=\gamma_{21 x}-R\left(\gamma_{20}+\gamma_{11}\right)+2 Q \gamma_{21}-5 \alpha_{1} \beta_{2}, \\
& \quad \delta_{30}=\gamma_{20 y}-S \gamma_{10}+Q \gamma_{21}-4 \alpha_{2} \beta_{1}-\alpha_{1} \beta_{2}, \\
& \epsilon_{10}=\delta_{10 x}-3 Q \delta_{10}+P\left(2 \delta_{20}+\delta_{11}\right)-12 \alpha_{0} \gamma_{10}, \\
& \lambda_{10}=\epsilon_{10 x}-4 Q \epsilon_{10}+P\left(3 \epsilon_{20}+\epsilon_{11}\right)-21 \alpha_{0} \delta_{10}, \\
& \alpha_{0}=Q_{x}-P_{y}+2 P R-2 Q^{2}, \quad \alpha_{1}=R_{x}-Q Q_{y}+P S-Q R, \\
& \alpha_{2}=S_{x}-R_{y}+2 Q S-2 R^{2} .
\end{aligned}
$$

In contrast to absolute invariants $I_{0}, I_{1}, I_{2}$, relative invariants are invariants not for the whole group of equivalence transformations of equation (1), but for some subgroup. A detailed description how to construct the relative and absolute invariants for equations (1) by means of Lie's infinitesimal approach can be found in [10]. We note that relations 12 and their differential consequences are satisfied for each equation of sixth type. Such trivial relations should be excluded from the necessary conditions of equivalence, since they do not reflect essential properties of a studied equation distinguishing it from other equations of the same type. At that, they play an essential role in the proof of sufficiency of equivalence conditions allowing us to express a part of invariants in terms of some "basic" invariants. This possibility is demonstrated in Section 5 in the proof of Theorem 7.

Remark. Equation (1) with $\beta_{1}=0, \beta_{2} \neq 0$ is reduced to equation with $\beta_{1} \neq 0$ by the hodograph transformation.

It was shown in [21] that each ODE (1) with relative invariants $J_{0}=0, j_{0}=0$ is reduced to (4) by some change of variables (2). Condition $j_{1}=0$ for such equation reads as

$$
\left(\frac{\partial^{3} f}{\partial y^{3}}\right)^{2}-\frac{5}{6} \frac{\partial^{2} f}{\partial y^{2}} \frac{\partial^{4} f}{\partial y^{4}}=0
$$


It implies that the right hand side of (4) is equal either to

$$
f(x, y)=b_{0}(x)+b_{1}(x) y+\frac{b_{4}(x)}{\left(y+b_{3}(x)\right)^{3}},
$$

or to

$$
f(x, y)=b_{0}(x)+b_{1}(x) y+b_{2}(x) y^{2} .
$$

Imposing other conditions in (9) for function $f(x, y)$ leads us to the conditions $b_{4}=$ const $\neq 0$, $b_{0}-b_{1} b_{3}+b_{3}^{\prime \prime} \neq 0$ for function (14) and to condition $b_{2} \neq 0$ for function (15). Under such restrictions, equation (4), (14) is reduced to (6) by an appropriate transformation (5), while equation (4), (15) is reduced to (7). The families of equations (6), (7) are non-equivalent since for the former we have $I_{1} \neq 0$, while the latter satisfies $I_{1}=0$. Necessary conditions of equivalence to equations of sixth type with $I_{1} \neq 0$ and $I_{1}=0$ are obtained in the subsequent sections by means of invariants $I_{0}, I_{1}, I_{2}, \mathcal{D}_{2}^{n} I_{2}, n \in \mathbb{N}$. It is easy to see that other derivatives of invariants (13) can be expressed by trivial relations 12 and their differential consequences in terms of invariants $I_{1}, I_{2}, \mathcal{D}_{2}^{n} I_{2}, n \in \mathbb{N}$.

\section{Equations of SiXth type With $I_{1} \neq 0$}

Equation (6) has the following invariants (here $\left.w^{\prime}=d w / d z\right)$

$$
\begin{aligned}
& I_{1}=5\left(1+w^{3} F\right), \\
& I_{2}=\frac{w^{10}\left(4 F^{2}-3 F F^{\prime \prime}\right)}{27\left(1+w^{3} F\right)^{2}}-\frac{w^{3} F}{36\left(1+w^{3} F\right)^{2}}\left(184 w^{6} F^{2}+389 w^{3} F+196\right), \\
& I_{22}=-\frac{w^{12}\left(9 F^{2} F^{\prime \prime \prime}-45 F F^{\prime} F^{\prime \prime}+40 F^{\prime 3}\right)}{27 \sqrt{3} F\left(1+w^{3} F\right)^{5 / 2}}, \quad I_{0}=-\frac{w\left(w F^{\prime}+3 w^{\prime} F\right)}{\sqrt{3} F\left(1+w^{3} F\right)^{3 / 2}} .
\end{aligned}
$$

Comparing invariants $I_{1}, I_{2}, I_{22}$, we see that the algebraic invariants

$$
\hat{J}_{1}=\frac{3^{9} \cdot 5^{4}}{\left(I_{1}-5\right)^{10}}\left(I_{1}^{2} I_{2}+\left(I_{1}-5\right)\left(\frac{46}{45} I_{1}^{2}+\frac{7}{12} I_{1}-\frac{5}{4}\right)\right)^{3}, \quad \hat{J}_{2}=\frac{3^{7} \cdot 5^{3} I_{1}^{5} I_{22}^{2}}{\left(I_{1}-5\right)^{8}}
$$

for equation (1) of sixth type with $I_{1} \neq 0$ depend on one variable only. In particular, for ODE (6) they are equal

$$
\hat{J}_{1}=-\frac{\left(3 F F^{\prime \prime}-4 F^{\prime 2}\right)^{3}}{F^{10}}, \quad \hat{J}_{2}=\frac{\left(9 F^{2} F^{\prime \prime \prime}-45 F F^{\prime} F^{\prime \prime}+40 F^{\prime 3}\right)^{2}}{F^{10}} .
$$

The condition $\hat{J}_{2}=0$ coincides with the existence of a point symmetry. Namely, ODE (6) admits the symmetry operator $X$ in the cases

1) $F(z)=$ const, $X=\partial_{z}$;

2) $F(z)=\frac{c}{z^{3 / 2}}, c=$ const $\neq 0, X=2 z \partial_{z}+w \partial_{w}$.

In the case $I_{1} \neq 0$, in obtaining necessary conditions of equivalence, it is reasonable to employ basis invariants $I_{0}, I_{1}$ and invariants 16 instead of invariants $I_{0}, I_{1}, I_{2}, I_{22}$. If $\hat{J}_{1}, \hat{J}_{2} \neq$ const for a given equation, then excluding the common variable in the expressions for $\hat{J}_{1}, \hat{J}_{2}$ gives a relation for $\hat{J}_{1}, \hat{J}_{2}$ being an invariant characteristic for this equation. Two equations with different relations for $\hat{J}_{1}, \hat{J}_{2}$ can not be equivalent.

In [25, Sect. 2.4.2] some equations (4) of sixth type with $I_{1} \neq 0$ were integrated. All of them are reduced to two ODEs. The necessary conditions of equivalence to these equations are formulated in the following statements.

Theorem 2. If equation (1) is equivalent to $O D E$

$$
\frac{d^{2} w}{d z^{2}}=\frac{A_{1}}{w^{3}}+A_{2}, \quad A_{1}, A_{2}=\text { const } \neq 0,
$$


it is an equation of sixth type, its invariants satisfy the conditions

$$
I_{1} \neq 0, \quad \hat{J}_{1}=0, \quad \hat{J}_{2}=0 .
$$

If equations (1), (17) are equivalent, change of variables (2) relating them is determined by the identities

$$
I_{1}=5+\frac{5 A_{2} w^{3}}{4 A_{1}}, \quad I_{0}=-\frac{4 \sqrt{3} A_{1} w w^{\prime}}{\left(-\left(4 A_{1}+A_{2} w^{3}\right)\right)^{3 / 2}} .
$$

Theorem 3. If equation (1) is equivalent to $O D E$

$$
\frac{d^{2} w}{d z^{2}}=\frac{A_{1}}{w^{3}}+\frac{A_{2}}{z^{3 / 2}}, \quad A_{1}, A_{2}=\text { const } \neq 0
$$

it is an equation of sixth type, its invariants satisfy the conditions

$$
I_{1} \neq 0, \quad \hat{J}_{1}=\text { const } \neq 0, \quad \hat{J}_{2}=0 .
$$

If equations (1), (20) are equivalent, change of variables (2) relating them and the relation between the parameters of the equations are determined by the identities

$$
\hat{J}_{1}=\frac{729 A_{1}}{16 A_{2}^{4}}, \quad I_{1}=5+\frac{5 A_{2} w^{3}}{4 A_{1} z^{3 / 2}}, \quad I_{0}=\frac{2 \sqrt{3} A_{1} z^{5 / 4} w\left(w-2 z w^{\prime}\right)}{\left(-\left(4 A_{1} z^{3 / 2}+A_{2} w^{3}\right)\right)^{3 / 2}} .
$$

If equations (1) and (17) (or (20) ) are equivalent, their invariants coincide, and thus, they satisfy the same relations. This is why the proof of the above theorem consists in straightforward calculating of invariants $I_{0}, I_{1}$ and $(16)$ for equations 170 and 20 .

\section{Equations of SiXth type With $I_{1}=0$}

Equation (7) have the following invariants

$$
I_{1}=0, \quad I_{2}=\frac{11}{2}+\frac{f}{108 w^{2}}, \quad \mathcal{D}_{2}^{n} I_{2}=\frac{f^{(n)}}{(-2)^{n} 108 w^{2+n / 2}}, \quad n \in \mathbb{N}, \quad I_{0}=-\frac{w^{\prime}}{6 w^{3 / 2}} .
$$

We see that the algebraic invariants

$$
\tilde{J}_{1}=\frac{I_{22}^{4}}{27\left(2 I_{2}-11\right)^{5}}, \quad \tilde{J}_{2}=\frac{I_{222}^{2}}{27\left(2 I_{2}-11\right)^{3}}
$$

depend on a single variable for equation (1) of sixth type $I_{1}=0, I_{2} \neq 11 / 2$. Thus, while obtaining necessary equivalence conditions for such equations of sixth type, it is useful to employ invariants (23) together with basis invariants (10). In particular, for ODE (7) with $f(z) \neq 0$ they read as

$$
\tilde{J}_{1}=\frac{\left(f^{\prime}\right)^{4}}{128 f^{5}}, \quad \tilde{J}_{2}=\frac{\left(f^{\prime \prime}\right)^{2}}{32 f^{3}} .
$$

Equation (7) admits symmetry operators if $2\left(2 I_{2}-11\right) I_{222}-5 I_{22}^{2}=0$, i.e., in the cases

1) $f(z)=0, X_{1}=\partial_{z}, X_{2}=z \partial_{z}-2 w \partial_{w}$;

2) $f(z)=$ const $\neq 0, X=\partial_{z}$;

3) $f(z)=\frac{c}{z^{4}}, c=$ const $\neq 0, X=z \partial_{z}-2 w \partial_{w}$.

Equations (4) of sixth type with $I_{1}=0$ considered in [25, Sect. 2.3.1, 2.4.2, 2.9.1] are reduced to equation (3), two ODES for which in [25, Sect. 2.3.1] the general solution was provided, and to an equation admitting the order reducing. The criterion of equivalence to the first Painlevé equation is proven in the next section, while necessary conditions of equivalence to other three equations in [25] are formulated in the following theorems. 
Theorem 4. If equation (1) is equivalent to $O D E$

$$
\frac{d^{2} w}{d z^{2}}=A w^{2}, \quad A=\text { const } \neq 0
$$

it is an equation of sixth type and its invariants satisfy the conditions

$$
I_{1}=0, \quad I_{2}=\frac{11}{2} .
$$

If equations (1), (24) are equivalent, change of variables (2) relating them is determined by the identity

$$
I_{0}=-\frac{w^{\prime}}{\sqrt{6 A} w^{3 / 2}}
$$

Theorem 5. If equation (1) is equivalent to $O D E$

$$
\frac{d^{2} w}{d z^{2}}=\frac{A w^{2}}{z^{5 / 2}}, \quad A=\text { const } \neq 0,
$$

it is an equation of sixth type and its invariants satisfy the conditions

$$
I_{1}=0, \quad I_{2} \neq \frac{11}{2}, \quad \tilde{J}_{1}=0, \quad \tilde{J}_{2}=0
$$

If equations (1), (27) are equivalent, change of variable (2) relating them is determined by the identities

$$
I_{2}=\frac{11}{2}-\frac{z}{18(\sqrt{z}+8 A w)^{2}}, \quad I_{0}=\frac{8 A z^{1 / 4}\left(w-2 z w^{\prime}\right)}{\sqrt{3}(\sqrt{z}+8 A w)^{3 / 2}} .
$$

Theorem 6. If equation (1) is equivalent to $O D E$

$$
\frac{d^{2} w}{d z^{2}}=\frac{3}{25}\left(\frac{w^{2}}{z^{3}}+\frac{2 c w}{z^{2}}\right), \quad c=\text { const } \neq \pm 1,
$$

it is an equation of sixth type and its invariants satisfy the conditions

$$
I_{1}=0, \quad I_{2} \neq \frac{11}{2}, \quad \tilde{J}_{1}=\text { const } \neq 0, \quad \tilde{J}_{2}=\text { const } \neq 0, \quad \frac{\tilde{J}_{2}}{\tilde{J}_{1}}=\frac{25}{4} .
$$

If equations (1), (30) are equivalent, change of variable (2) relating them is determined by the identities

$$
\begin{aligned}
\tilde{J}_{1} & =\frac{4}{3\left(1-c^{2}\right)}, \quad I_{2}=\frac{11}{2}+\frac{\left(1-c^{2}\right) z^{2}}{18((c+1) z+w)^{2}} \\
I_{0} & =\frac{\sqrt{z}\left(3 w-2(c+1) z-5 z w^{\prime}\right)}{3 \sqrt{2}((c+1) z+w)^{3 / 2}} .
\end{aligned}
$$

\section{CRiterion of equivalence to first Painlevé equation}

An invariant characteristic (necessary equivalence conditions) in terms of invariants (10), (13) for equation (3) was obtained in [10]. Here we prove the sufficiency of these conditions.

Theorem 7. Equation (1) is equivalent to the first Painlevé equation if and only if it is an equation of sixth type and its invariants satisfy the conditions

$$
I_{1}=1, \quad I_{222}=0, \quad \frac{\partial\left(I_{2}, I_{22}\right)}{\partial(x, y)} \neq 0 .
$$

Change of variables (2) reducing it to equation (3) is determined by the identities

$$
w^{5}=\frac{1}{6^{6} I_{22}^{2}}, \quad \frac{z}{w^{2}}=54\left(2 I_{2}-11\right) .
$$


Proof. To the prove the necessity, let us find the relations to be satisfied by the invariants of equation (3) and therefore, the same relations are to be satisfied by the invariants of equation (1) equivalent to ODE (3). Basis algebraic invariants (10), operators (11) and the invariants $\mathcal{D}_{2}^{n} I_{2}, n=1,2$, for ODE (3) are equal to

$$
\begin{aligned}
& I_{1}=0, \quad I_{2}=\frac{11}{2}+\frac{z}{108 w^{2}}, \quad \mathcal{D}_{1}=-3 w \partial_{w}, \quad \mathcal{D}_{2}=-\frac{1}{2 \sqrt{w}} \partial_{z}, \\
& I_{22}=-\frac{1}{216 w^{5 / 2}}, \quad I_{222}=0 .
\end{aligned}
$$

Invariants (35) satisfy first two identities in (33) and the condition $\partial\left(I_{2}, I_{22}\right) / \partial(z, w) \neq 0$, which is invariant w.r.t. a non-degenerate change of variables. The expressions for $I_{2}, I_{22}$ can be resolved w.r.t. $w^{5}, z w^{-2}$ that gives 34 ).

To probe the sufficiency, let us show that once conditions (33) for equation (1) are satisfied, the change of variables determined by (34) transforms it into equation (3). Differentiating (34) twice, for the derivatives of $w$ w.r.t. $z$ we obtain the expressions

$$
\begin{aligned}
& w \frac{d w}{d z}=\frac{\Omega}{54\left(2\left(2 I_{2}-11\right) \Omega-5 I_{22}\right)}, \\
& w^{3} \frac{d^{2} w}{d z^{2}}=\frac{4\left(11-2 I_{2}\right) \Omega^{3}+5 I_{22} \Omega^{2}+25 I_{22}^{2} d \Omega / d I_{2}}{18^{3}\left(2\left(2 I_{2}-11\right) \Omega-5 I_{22}\right)^{3}},
\end{aligned}
$$

where

$$
\Omega=\frac{d I_{22}}{d I_{2}}=\frac{\partial_{x} I_{22}+y^{\prime} \partial_{y} I_{22}}{\partial_{x} I_{2}+y^{\prime} \partial_{y} I_{2}}, \quad \frac{d \Omega}{d I_{2}}=\frac{\partial_{x} \Omega+y^{\prime} \partial_{y} \Omega+y^{\prime \prime} \partial_{y^{\prime}} \Omega}{\partial_{x} I_{2}+y^{\prime} \partial_{y} I_{2}} .
$$

Equation (3) can be represented as

$$
w^{3} \frac{d^{2} w}{d z^{2}}=w^{5}\left(6+\frac{z}{w^{2}}\right) .
$$

By substituting (34), 36) we transform it into

$$
50 I_{22}^{4} \frac{d \Omega}{d I_{2}}+8\left(11-2 I_{2}\right) I_{22}^{2} \Omega^{3}+10 I_{22}^{3} \Omega^{2}+3\left(49-9 I_{2}\right)\left(2\left(2 I_{2}-11\right) \Omega-5 I_{22}\right)^{3}=0 .
$$

By the identities $I_{1 j}=\mathcal{D}_{1} I_{j}, I_{2 j}=\mathcal{D}_{2} I_{j}$, where $I_{j}=I_{2}$ and $I_{j}=I_{22}$, while $\mathcal{D}_{1}, \mathcal{D}_{2}$ are determined in (11), we find the derivatives

$$
\begin{array}{ll}
\partial_{x} I_{j}=\frac{1}{3}\left(M_{2} I_{1 j}-M_{1} I_{2 j}\right), & \partial_{y} I_{j}=\frac{1}{3}\left(M_{4} I_{1 j}-M_{3} I_{2 j}\right), \\
M_{1}=\beta_{1} \sqrt{j_{3}}, \quad M_{2}=\frac{15 \beta_{1} e_{0}}{j_{3}\left(I_{1}-5\right)}, & M_{3}=\frac{\beta_{2}}{\beta_{1}} M_{1}, \quad M_{4}=\frac{\beta_{2}}{\beta_{1}} M_{2}-\frac{3 \beta_{1}}{M_{1}^{2}} .
\end{array}
$$

Second expression (37) depends also on the derivatives

$$
\begin{aligned}
\partial_{x}^{2} I_{j}= & \frac{1}{9}\left(M_{2}^{2} I_{11 j}-M_{1} M_{2}\left(I_{12 j}+I_{21 j}\right)+M_{1}^{2} I_{22 j}\right)+\frac{1}{3}\left(M_{2 x} I_{1 j}-M_{1 x} I_{2 j}\right), \\
\partial_{x} \partial_{y} I_{j}= & \frac{1}{9}\left(M_{2}\left(M_{4} I_{11 j}-M_{3} I_{21 j}\right)+M_{1}\left(M_{3} I_{22 j}-M_{4} I_{12 j}\right)\right) \\
& +\frac{1}{3}\left(M_{2 y} I_{1 j}-M_{1 y} I_{2 j}\right), \\
\partial_{y}^{2} I_{j}= & \frac{1}{9}\left(M_{4}^{2} I_{11 j}-M_{3} M_{4}\left(I_{12 j}+I_{21 j}\right)+M_{3}^{2} I_{22 j}\right)+\frac{1}{3}\left(M_{4 y} I_{1 j}-M_{3 y} I_{2 j}\right) .
\end{aligned}
$$


The derivatives of the first two functions in $(40)$ are equal to

$$
\begin{aligned}
M_{1 x}= & \left(\frac{I_{1}-5}{10} M_{2}+\frac{2 \gamma_{10}}{5 \beta_{1}}-P \frac{\beta_{2}}{\beta_{1}}+Q\right) M_{1}, \\
M_{1 y}= & \left(\frac{I_{1}-5}{10} M_{4}+\frac{2 \gamma_{20}-3 \gamma_{11}}{5 \beta_{1}}+\frac{3 \beta_{2} \gamma_{10}}{5 \beta_{1}^{2}}-Q \frac{\beta_{2}}{\beta_{1}}+R\right) M_{1}, \\
M_{2 x}= & \left(15-2 I_{1}\right) \frac{M_{2}^{2}}{15}+\frac{5 M_{1}^{2}\left(49\left(5-I_{1}\right)-45 I_{2}\right)}{3\left(I_{1}-5\right)^{2}}+\left(\frac{2 \gamma_{10}}{5 \beta_{1}}+Q\right) M_{2}-P M_{4}, \\
M_{2 y}= & \left(15-2 I_{1}\right) \frac{M_{2} M_{4}}{15}+\frac{5 M_{1} M_{3}}{3\left(I_{1}-5\right)^{2}}\left(49\left(5-I_{1}\right)-45 I_{2}\right) \\
& +\left(\frac{\gamma_{20}+\gamma_{11}}{5 \beta_{1}}-\frac{\beta_{2} \gamma_{10}}{5 \beta_{1}^{2}}+R\right) M_{2}+\left(\frac{\gamma_{10}}{5 \beta_{1}}-Q\right) M_{4},
\end{aligned}
$$

while the derivatives $M_{3 y}, M_{4 y}$ are calculated by means of the expressions for $M_{1 y}, M_{2 y}$ and $\beta_{1 y}=\gamma_{20}+R \beta_{1}-Q \beta_{2}, \beta_{2 y}=\gamma_{21}+S \beta_{1}-R \beta_{2}$.

Substituting quantities (37), where the derivatives of invariants $I_{2}, I_{22}$ are calculated by means of (39), (41), and $y^{\prime \prime}$ is replaced by means of (1), we cast (38) into the form

$$
\begin{aligned}
\Lambda_{0}\left(M_{2}+y^{\prime} M_{4}\right)^{3} & +3 \Lambda_{1}\left(M_{1}+y^{\prime} M_{3}\right)\left(M_{2}+y^{\prime} M_{4}\right)^{2} \\
& +3 \Lambda_{2}\left(M_{1}+y^{\prime} M_{3}\right)^{2}\left(M_{2}+y^{\prime} M_{4}\right)+\Lambda_{3}\left(M_{1}+y^{\prime} M_{3}\right)^{3}=0
\end{aligned}
$$

with coefficients $\Lambda_{i}$ being functions of the invariants for equation (1). Identity (42) is the condition ensuring that equations (1) and (3) are related by the change of variables determined by (34). It remains to show that $\Lambda_{i}$ vanishes if the invariants of equation (1) satisfy conditions (33).

By trivial identities (12) and their consequences, we can express invariants $I_{12}$ and

$$
\begin{aligned}
& I_{112}=\frac{4}{25}\left(7 I_{1}^{2}+45 I_{1}+225\right) I_{2}+\frac{I_{1}-5}{225}\left(92 I_{1}^{2}+2217 I_{1}+8910\right), \\
& I_{122}=\frac{3}{10}\left(3 I_{1}+25\right) I_{22}, \quad I_{1122}=\frac{9}{20}\left(3 I_{1}^{2}+24 I_{1}+125\right) I_{22}, \\
& I_{212}=\frac{2}{5}\left(2 I_{1}+15\right) I_{22}, \quad I_{2122}=\frac{3}{10}\left(3 I_{1}+25\right) I_{222}, \quad I_{1222}=\left(I_{1}+9\right) I_{222}
\end{aligned}
$$

in terms of invariants $I_{1}, I_{2}, I_{22}, I_{222}$. Substituting these expressions into $\Lambda_{i}$, we obtain

$$
\begin{aligned}
\Lambda_{0}= & 2 I_{1} I_{22}^{3}\left[4 I_{1}^{2}\left(9 I_{2}-49\right) K_{0}^{3}-81 I_{1}\left(33 I_{1}+875\right) I_{22}^{2} K_{0}\right. \\
& \left.+243\left(3 I_{1}+25\right)\left(23 I_{1}^{2}+8460 I_{1}+56125\right) I_{22}^{2}\right] \\
\Lambda_{1}= & 30 I_{1} I_{22}^{2}\left[6 I_{1}\left(9 I_{2}-49\right) K_{0}^{2} K_{1}-243\left(9 I_{1}+175\right) I_{22}^{2} K_{1}\right. \\
& \left.+\left(13 K_{2}-10\left(17 I_{1}+105\right) K_{0}-375\left(343 I_{1}+2245\right)\right) I_{22}^{2} I_{222}\right], \\
\Lambda_{2}= & 225 I_{22}\left[18 I_{1}\left(9 I_{2}-49\right) K_{0} K_{1}^{2}-162\left(3 I_{1}+25\right) I_{22}^{2} I_{222} K_{1}\right. \\
& \left.-45\left(431 I_{1}+3525\right) I_{22}^{4} I_{222}+2 I_{22}^{2}\left(I_{222}^{2}+45 I_{22} I_{2222}\right) K_{2}\right] \\
\Lambda_{3}= & 15^{3}\left[27\left(9 I_{2}-49\right) K_{1}^{3}+36 I_{22}^{2} I_{222}^{2} K_{1}+90 I_{22}^{4}\left(46 I_{222}^{2}-45 I_{22} I_{2222}\right)\right. \\
& \left.+5 I_{22}^{4}\left(I_{1}-5\right)^{-2}\left(49\left(I_{1}-5\right)+45 I_{2}\right)\left(135\left(3 I_{1}+25\right) I_{22}^{2}-2 I_{222} K_{2}\right)\right],
\end{aligned}
$$

where $K_{0}=9 I_{2}+23 I_{1}+1073, K_{1}=2\left(2 I_{2}-11\right) I_{222}-5 I_{22}^{2}, K_{2}=45\left(2 I_{1}+15\right)\left(2 I_{2}-11\right)+$ $I_{1}\left(46 I_{1}+2245\right)$. It can be shown that these quantities vanish if and only if $I_{1}=0, I_{222}=0$, $I_{2222}=0$. The proof is complete. 


\section{EXAMPLES OF EQUIVALENT EQUATIONS}

Apart from equations (17), 20), (24), (27), in [25] there were integrated other ODEs (4) of sixth type equivalent to these four equations. In two subsequent examples we show how to establish the equivalence of equation by means of invariants and to find the relating change of variables (2). In the third example we establish that the generalized Sundman transform obtained in [20], up to a point change of variables can be regarded as autotransformation of the first Painlevé equation.

Example 1. In [25], there was provided the general solution to ODE

$$
\frac{d^{2} y}{d x^{2}}=\frac{A_{1}}{y^{3}}+\frac{A_{2}}{x^{3}}, \quad A_{1}, A_{2}=\text { const } \neq 0,
$$

having invariants

$$
I_{1}=5+\frac{5 A_{2} y^{3}}{4 A_{1} x^{3}}, \quad I_{0}=\frac{4 \sqrt{3} A_{1} x^{7 / 2} y\left(y-x y^{\prime}\right)}{\left(-\left(4 A_{1} x^{3}+A_{2} y^{3}\right)\right)^{3 / 2}}, \quad \hat{J}_{1}=0, \quad \hat{J}_{2}=0,
$$

satisfying conditions (18) of Theorem 2. Equating invariants $I_{1}$ defined by (19), 44), we obtain the transformation relating equations (43) and (17):

$$
z=\xi(x, y), \quad w=\frac{y}{x}, \quad \frac{d w}{d z}=\frac{x y^{\prime}-y}{x^{2}\left(\xi_{x}+y^{\prime} \xi_{y}\right)} .
$$

Equating invariants $I_{0}$ and substituting (45), we obtain the equations $\xi_{x}=x^{-2}, \xi_{y}=0$ to determine function $\xi$. The solution is $\xi=-1 / x$. It is easy to check that the change of variables $z=-1 / x, w=y / x$ transforms (43) into equation (17).

Example 2. In [25] there was integrated the equation

$$
\frac{d^{2} y}{d x^{2}}=\frac{A y^{2}}{x^{2}}-\frac{6 y}{25 x^{2}}, \quad A=\text { const } \neq 0
$$

whose invariants are

$$
I_{1}=0, \quad I_{2}=\frac{11}{2}, \quad I_{0}=\frac{2 y-5 x y^{\prime}}{5 \sqrt{6 A} y^{3 / 2}} .
$$

Comparing (47) with (25), (28), (31), we conclude that ODE (46) satisfies the hypothesis of Theorem 4 and is equivalent to equation (24). Change (2) relating (46) and (24) is found by the condition of equality of invariants $I_{0}$ for these equations. Substituting

$$
z=\xi(x, y), \quad w=\eta(x, y), \quad \frac{d w}{d z}=\frac{\eta_{x}+y^{\prime} \eta_{y}}{\xi_{x}+y^{\prime} \xi_{y}}
$$

into this identity and equating the coefficients at the like powers of $y^{\prime}$, for $\xi, \eta$ we obtain the system of equations

$$
5 \sqrt{y} \eta_{x}+2 \eta^{3 / 2} \xi_{x}=0, \quad 5 y^{3 / 2} \eta_{y}+\eta^{3 / 2}\left(2 y \xi_{y}-5 x \xi_{x}\right)=0, \quad \xi_{y}=0 .
$$

One of its solutions is $\xi=5 x^{1 / 5}, \eta=y x^{-2 / 5}$ and thus, ODE (46) is transformed to (24) by the change of variables $z=5 x^{1 / 5}, w=y x^{-2 / 5}$.

Example 3. It was shown in [20] that the generalized Emden-Fowler equation

$$
\frac{d^{2} y}{d x^{2}}=\frac{c_{0} y^{2}}{(x-k)^{5}}+\frac{c_{1}}{(x-k)^{4}}+\frac{c_{2}}{(x-k)^{3}}, \quad c_{0}, c_{1}, c_{2}, k=\mathrm{const}, \quad c_{0}, c_{1} \neq 0
$$

is related with the first Painlevé equation

$$
\frac{d^{2} W}{d Z^{2}}=6 W^{2}-\frac{625}{6} Z
$$


by the generalized Sundman transform

$$
Z=-\frac{\left(c_{0} c_{1}\right)^{1 / 5}}{5^{4 / 5}}\left(\frac{1}{x-k}+\frac{c_{2}}{c_{1}}\right), \quad U(Z)=\frac{\left(5 c_{0} c_{1}\right)^{4 / 5}}{6 c_{1}} \int \frac{y(x)}{(x-k)^{3}} d x,
$$

where $d U(Z) / d Z=W(Z)$. By the dilatation $Z=-5^{-4 / 5} 6^{1 / 5} z, W=5^{8 / 5} 6^{-2 / 5} w$, the Painlevé equation is reduced to the standard form (3). ODE (48) is an equation of sixth type. Its algebraic invariants

$$
I_{1}=0, \quad I_{2}=\frac{11}{2}+\frac{(x-k)\left(c_{1}+c_{2}(x-k)\right)}{18 c_{0} y^{2}}, \quad I_{22}=\frac{c_{1}(x-k)^{5 / 2}}{\left(6 c_{0}\right)^{3 / 2} y^{5 / 2}}, \quad I_{222}=0
$$

satisfy conditions (33) in Theorem 7 . Therefore, equation (48) is equivalent to the first Painlevé equation. The corresponding transformation

$$
z=\frac{\left(c_{0} c_{1}\right)^{1 / 5}}{6^{1 / 5}}\left(\frac{1}{x-k}+\frac{c_{2}}{c_{1}}\right), \quad w=\frac{c_{0}^{3 / 5} y}{6^{3 / 5} c_{1}^{2 / 5}(x-k)}
$$

is determined by relations (34), which in the present case read as

$$
w^{5}=\frac{c_{0}^{3} y^{5}}{216 c_{1}^{2}(x-k)^{5}}, \quad \frac{z}{w^{2}}=\frac{6(x-k)\left(c_{1}+c_{2}(x-k)\right)}{c_{0} y^{2}} .
$$

Thus, equation (48) is related with the first Painlevé equation not only by nonlocal transformation (49), but also by a simpler change of variables (50).

\section{BIBLIOGRAPHY}

1. R. Liouville. Mémoire sur les invariants de certaines équations différentielles et sur leurs applications // J. École Polytechnique. 59, 7-76 (1889).

2. A. Tresse. Sur les invariants différentiels des groupes continus de transformations // Acta Math. 18:1, 1-88 (1894).

3. É. Cartan Sur les variétés à connexion projective // Bull. Soc. Math. France. 52:205-241 (1924).

4. G. Thomsen. Über die topologischen Invarianten der Differentialgleichung $y^{\prime \prime}=f(x, y) y^{\prime 3}+$ $g(x, y) y^{\prime 2}+h(x, y) y^{\prime}+k(x, y) / /$ Abh. Math. Semin. Hamb. Univ. 7:1, 301-328 (1929).

5. V.V. Dmitrieva, R.A. Sharipov. On the point transformations for the second order differential equations. I // Preprint arXiv:solv-int/9703003 (1997).

6. R.A. Sharipov. On the point transformations for the equations $y^{\prime \prime}=P+3 Q y^{\prime}+3 R y^{\prime 2}+S y^{\prime 3} / /$ Bull. Bashkir State University. 1:1, 5-8 (1998) [Preprint arXiv:solv-int/9706003 (1997).]

7. R.A. Sharipov. Effective procedure of point classification for the equations $y^{\prime \prime}=P+3 Q y^{\prime}+3 R y^{\prime 2}+S y^{\prime 3} / /$ Preprint arXiv:math.DG/9802027 (1998).

8. V.A. Yumaguzhin. Differential invariants of second order ODEs. I // Acta Appl. Math. 109:1, 283-313 (2010).

9. O.I. Morozov. Problem of point equivalence for second order ordinary differential equations. II // Scientific bull. MSTU SA 157 98-104 (2010). (in Russian).

10. Yu.Yu. Bagderina. Invariants of a family of scalar second-order ordinary differential equations // J. Phys. A. 46:29, id 295201, 36pp (2013).

11. E.L. Ince. Ordinary differential equations. Dover Publications, New York (1926).

12. P. Painlevé. Sur les équations différentielles du second ordre et d'ordre supérieur dont l'intégrale générale est uniforme // Acta Math. 25:1, 1-85 (1902).

13. B. Gambier. Sur les équations différentielles du second ordre et du premier degré dont l'intégrale générale est à points critiques fixes // Acta Math. 33:1, 1-55 (1910).

14. N. Kamran, K.G. Lamb, W.F. Shadwick. The local equivalence problem for $d^{2} y / d x^{2}=F(x, y, d y / d x)$ and the Painlevé transcendents // J. Diff. Geom. 22:2, 139-150 (1985). 
15. N. Kamran, W.F. Shadwick. A differential geometric characterization of the first Painlevé transcendent// Math. Ann. 279:1, 117-123 (1987).

16. V.V. Dmitrieva. On classification of equations Painlevé-I and Painlevé-ii under general point trasnformations // Bull. Bashkir State University. 1:1, 9-11 (1998). (in Russian).

17. A.V. Bocharov, V.V. Sokolov, S.I. Svinolupov. On some equivalence problems for differential equations // Preprint ESI 54, 12pp (1993).

18. R. Dridi. On the geometry of the first and second Painlevé equations // J. Phys. A. 42:12, id 125201, 9pp (2009). V. 42. (9pp).

19. V.V. Kartak. Explicit solution of the equivalence problem for certain Painlevé equations // Ufimskij Matem. Zhurn. 1:3, 46-56 (2009). (in Russian).

20. M. Euler, N. Euler, A. Strömberg, E. Âström. Transformation between a generalized EmdenFowler equation and the first Painlevé transcendent // Math. Meth. Appl. Sci. 30:16, 2121-2124 (2007).

21. M.V. Babich, L.A. Bordag. Projective differential geometrical structure of the Painlevé equations // J. Differ. Equat. 157:2, 452-485 (1999).

22. Yu. Yu. Bagderina. Equivalence of second-order ordinary differential equations to Painlevé equations // Teor. Matem. Fiz. 182:2, 256-276 (2015). [Theor. Math. Phys. 182:2, 211-230 (2015).]

23. J. Hietarinta, V. Dryuma. Is my ODE a Painlevé equation in disguise? // J. Nonlin. Math. Phys. 9:Suppl. 1, 67-74 (2002).

24. Yu.Yu. Bagderina, N.N. Tarkhanov. Solution of the equivalence problem for the third Painlevé equation // J. Math. Phys. 56:1, id 013507, 15pp (2015).

25. V.F. Zajtsev, A.D. Polyanin. Handbook of ordinary differential equations. Fizmatlit, Moscow (2001). (in Russian).

26. R. Milson, F. Valiquette. Point equivalence of second-order ODEs: maximal invariant classification order // J. Symb. Comput. 67, 16-41 (2015).

Yulia Yurievna Bagderina, Institute of Mathematics CC USC RAS, Chernyshevsky str., 112, 450008, Ufa, Russia

E-mail: yulya@mail.rb.ru 